whom 53\% $(\mathrm{n}=8)$ had a completed bundle. 9 ascitic taps were attempted in 13 patients with ascites (69\%); correct investigations were requested in 5 of 7 taps (71\%). At a repeat online survey (32\% response rate) 10\% (3/30) selected only the necessary investigations for new ascites and 13\% (4/ 30) for recurrent ascites. This indicates that knowledge has not significantly improved.

Conclusion There remained low usage of the BSG-BASL decompensated cirrhosis bundle despite an education campaign. Ascitic tap performance improved but test requesting remained suboptimal. Systematic changes such as IT-system alerts and integrated guidance on key ascites tests within bundle documentation may be more sustainable ways of improving compliance (data will be presented).

\section{P043 EXPLORATORY PROTEOMIC ANALYSIS OF SYSTEMIC CHANGES IN ALF PATIENTS UNDERGOING PEX}

\author{
1,2Benoit Jauniaux*, ${ }^{2}$ Roz Banks, ${ }^{1}$ Marina Karakantza, ${ }^{1}$ Maria Kerr, ${ }^{1}$ Niki Snook, \\ ${ }^{1}$ Mark Bellamy, 'Joanna Moore. 'Leeds Teaching Hospitals Trust, St James's Hospital, UK; \\ ${ }^{2}$ University of Leeds, Leeds, UK
}

10.1136/gutjnl-2021-BASL.52

Background Acute liver failure (ALF) is defined by hepatic encephalopathy and an International Normalised Ratio greater than 1.5 in a patient with no underlying chronic liver disease. Standard-volume Plasma Exchange (SVPEX) is an extracorporeal procedure that separates plasma constituents from the cellular blood components by centrifugation and replaces it with stored plasma. There is mounting evidence supporting the clinical use of PEX as a therapy in ALF. The primary objective of this prospective pilot-exploratory proteomic study is to investigate the broad systemic changes induced by PEX on circulating proteins in patients with ALF before and after PEX.

Methods Clinical parameters were compared in paracetamolinduced ALF patients receiving $\operatorname{PEX}(n=3)$ and ALF patients not receiving PEX $(n=4)$. Circulating plasma protein levels were compared via mass spectrometry in 17 plasma samples from patients receiving $\operatorname{PEX}(n=2)$, not receiving $\operatorname{PEX}(n=2)$ and healthy controls $(n=2)$.

Results INR and PT medians were 10\% higher at baseline in the PEX group compared to ALF controls. Following the first PEX cycle, INR and PT were 20\% lower than in ALF controls on day 2. On mass spectrometry, several circulating proteins increased over two-fold immediately or 12 hours following PEX, in particular clotting and complement factors and apolipoproteins. Some proteins decreased to less than $50 \%$ following PEX, with the $20 \mathrm{~S}$ proteasomes being of particular interest.

Discussion This study is the first broad-spectrum proteomic analysis of plasma proteins in patients with ALF during and after SVPEX. PEX may improve patient clinical parameters and prognosis by several mechanisms, including the removal of the $20 \mathrm{~S}$ proteasomes of the ubiquitin pathway, which may have a role in inducing vascular damages and multi-organ failure. This initial analysis has highlighted the potential of proteomics to profile changes induced in PEX. This will now be extended and validated with additional patients and proteomic approaches by the end of July.

\section{P044 HEALTHCARE RECOURSE USE IN PATIENTS WITH CIRRHOTIC ASCITES: A UK REAL WORLD EVIDENCE STUDY}

${ }^{1}$ Kris Bennett*, ${ }^{2}$ Elisabet Viayna, ${ }^{3}$ Duncan Stacey, ${ }^{4}$ David Southern, ${ }^{1}$ Matthew Cramp. ${ }^{1}$ University Hospitals Plymouth NHS Trust, Plymouth, UK; ${ }^{2}$ Grifols S.A, Sant Cugat del Vallès, Spain; ${ }^{3}$ Grifols UK, Cambridge, UK; ${ }^{4}$ Spirit Access Ltd., Leicester, UK

\subsection{6/gutjnl-2021-BASL.53}

Introduction Decompensated cirrhosis is a major cause of morbidity and mortality in the United Kingdom (UK). ${ }^{1}$ The development of ascites complicating cirrhosis frequently represents the first manifestation of decompensation, is a hallmark of advanced liver disease and frequently a prelude to other complications. Refractory ascites (RA), characterised by early recurrence after initial paracentesis, is associated with a worse prognosis. $^{2}$ The objective of the present study was to describe healthcare resource utilisation and costs incurred by cirrhotic patients following presentation to the South West Liver Unit with large volume ascites requiring paracentesis.

Methods A retrospective study using the Hospital Episode Statistics (HES) data from University Hospitals Plymouth was performed. Patients with decompensated cirrhosis and ascites based on liver disease ICD-10 codes (K70-K77) requiring paracentesis (T46) between January-2015 and February-2020 were identified and healthcare resource utilisation/costs assessed over the following 12 months or until Transjugular Intrahepatic Portosystemic Shunt (TIPS) insertion, liver transplant, diagnosis of RA or death. RA was defined by the need for 2 or more paracenteses in a month. The subset of patients who developed RA were followed up for 12 additional months. Outpatient visits, elective and non-elective admissions and mortality were quantified and costed for the overall population and the sub-group who developed RA.

Results HES data identified a cohort of 524 cirrhotic patients with ascites. Mean age was 61.8 years and $57.8 \%$ were male. 12-month mortality was 22.3\%. 3489 outpatient visits, 509 elective day cases, 284 elective admissions and 969 non-elective admissions were required. The mean cost per patient was $£ 12,176$ per year. A total of 1061 paracenteses were performed during the 12-month follow-up. RA developed in 129 $(24.6 \%)$ cases. Patients with RA were more likely to require hospitalisation (421 day cases, 421 non-elective and 206 elective admissions), had more outpatient appointments (1693) and a greater requirement for paracenteses (618 procedures) during the subsequent 12 months. This resulted in a greater mean cost per patient ( $£ 23,810$ per year).

Conclusions The development of ascites in cirrhotic patients is associated with significant healthcare resource utilisation and high mortality. One in four patients developed refractory ascites within 12 months of first paracentesis and in these patients hospitalisation rates, outpatient appointments and paracentesis more than doubled, with a significant increase in associated cost. Future treatment strategies that can reduce the likelihood of developing RA may help mitigate costs and ease the burden on both health systems and patients.

\section{REFERENCES}

1. Sepanlou SG, Safiri S, Bisignano C, et al. The global, regional, and national burden of cirrhosis by cause in 195 countries and territories, 1990-2017: a systematic analysis for the Global Burden of Disease Study 2017. The Lancet Gastroenterology \& Hepatology 2020;5(3):245-66. 